\title{
Evaluation of the Spermatogenic Activity of Polyherbal Formulation in Oligospermic Males
}

\author{
Syed Ammar Hussain $\mathbb{D D}^{1}{ }^{1}$ Ahsan Hameed, ${ }^{1}$ Furqan Nasir, ${ }^{2}$ Yang Wu, \\ Hafiz Ansar Rasul Suleria $\left(\mathbb{1}^{3},{ }^{3,4,5}\right.$ and Yuanda Song $\mathbb{1}^{1}$ \\ ${ }^{1}$ Colin Ratledge Center for Microbial Lipids, School of Agriculture Engineering and Food Science, \\ Shandong University of Technology, Zibo 255049, China \\ ${ }^{2}$ Mayo Hospital Affiliated with King Edward Medical University, Lahore, Pakistan \\ ${ }^{3}$ UQ Diamantina Institute, Translational Research Institute, Faculty of Medicine, The University of Queensland, \\ 37 Kent Street Woolloongabba, Brisbane, QLD 4102, Australia \\ ${ }^{4}$ Department of Food, Nutrition, Dietetics and Health, Kansas State University, Manhattan, KS 66506, USA \\ ${ }^{5}$ Centre for Chemistry and Biotechnology, School of Life and Environmental Sciences, Deakin University, Pigdons Road, \\ Waurn Ponds, VIC 3216, Australia
}

Correspondence should be addressed to Hafiz Ansar Rasul Suleria; hafiz.suleria@uqconnect.edu.au and Yuanda Song; ysong@sdut.edu.cn

Received 1 January 2018; Accepted 29 May 2018; Published 25 July 2018

Academic Editor: Berkan Resorlu

Copyright (c) 2018 Syed Ammar Hussain et al. This is an open access article distributed under the Creative Commons Attribution License, which permits unrestricted use, distribution, and reproduction in any medium, provided the original work is properly cited.

The therapeutic use of natural herbs is an ancient human civilization act and the numbers of people have reliance on their pharmacological properties and preferred to use the natural herbs. People also use to consume these herbs as supplements to energize, bolster, and eventually enhance sexual ability. Polyherbal formulation (PHF) is one of these herbal amalgams that can be used to treat sexual dysfunction including erectile dysfunction, impotence, ejaculation dysfunction, and hypogonadism. The pilot study was aimed at evaluating the capacity of PHF in enhancing the spermatogenic potential of oligospermic patients. Thirty-six male patients with oligospermia were enrolled and randomized either to treatment $(n=23)$ with PHF $(750 \mathrm{mg} / \mathrm{d}$ in three doses for 90 days) or to placebo $(n=13)$ in the same protocol. The preintervention semen analysis was compared with posttreatment semen analysis. Based on the postintervention semen analysis, patients were advised to undergo either in vitro fertilization (IVF) or intracytoplasmic sperm injection (ICSI) to assess their fertility status. After polyherbal treatment, there was a $256 \%$ increase in sperm concentration $\left(9.59 \pm 4.37 \times 106 / \mathrm{mL}\right.$ to $\left.25.61 \pm 8.6 \times 10^{6} / \mathrm{mL} ; \mathrm{P} \leq 0.001\right), 154 \%$ increase in semen volume $(1.7 \pm 0.14 \mathrm{~mL}$ to $4.32 \pm 0.38 \mathrm{~mL} ; \mathrm{P} \leq 0.001)$, and $215 \%$ increase in sperm motility $(15.43 \pm 2.40 \%$ to $48.65 \pm 5.10 \%$; $\leq 0.001)$ on day 90 from baseline. Furthermore, a significant improvement and regulation were also observed in serum hormone levels with PHF treatment as compared to the placebo group. The present study demonstrated the evidence on synergistic spermatogenic effect of PHF as attributed in ayurveda for the treatment of oligospermia leading to infertility.

\section{Introduction}

Among human infertility, male infertility accounts for almost $50 \%$. Among $40 \%$ to $50 \%$ of these infertile males, the etiologies remain unidentified [1-7]. Approximately $8 \%$ of these infertile men are looking forward to medical treatment to resolve infertility related issues [8]. Male infertility is caused by a wide range of etiologies. Numerous factors are defined with unequivocal and harmful effect on male reproduction function, i.e., (i) modern (sedentary) life style problems like obesity, smoking, caffeine, alcohol, and drug abuse, (ii) nutritional deficiencies including vitamin and mineral deficiencies [9] and oxidative stress [10], (iii) genetic causes related to male infertility like the cystic fibrosis transmembrane conductance regulator (CFTR) gene mutations leading to congenital absence of the vas deferens, 
Y-chromosome microdeletions in the azoospermia factor locus leading to spermatogenic impairment, and karyotype abnormalities, oncological diseases, (iv) hormonal issues encompass: hyperprolactinemia, hypothyroidism, congenial adrenal hyperplasia, hypogonadotropic hypogonadism, and panhypopituitarism, (v) physical problems likewise varicocele, damaged sperm ducts, torsion, Klinefelter's syndrome, retrograde ejaculation, psychological issues comprised of erectile dysfunction, premature ejaculation, and ejaculatory incompetence [11, 12]; hence, different approaches are required to resolve all these multifaceted dilemmas [13]. Oligospermia may be considered as a pathologic effect of the above-mentioned causes and consequently oligospermia represents a cause of reduced male fertility $[1,2]$.

Broad range of strategies had been carried out to overcome the male infertility complications. Human chorionic gonadotropin (hCG) [14], treatment of follicle-stimulating hormone (FSH) [15], and usage of assisted reproduction technology (ART) are of the state-of-the-art options for these infertility issues [16]. However, shortcomings of the above stated treatments cannot be overlooked, which may include acceptability, high expenditure, and threat of diverse complications during surgical procedures [17]. Approximately half of the couples undergoing consultation for male infertility terminated ART treatment, mainly due to the physical and emotional pain resulting from the treatment [16]. Additionally, there were some concerns for the offspring conceived by means of ART, including in vitro fertilization (IVF) and intracytoplasmic sperm injection (ICSI) via microsurgical epididymal sperm aspiration (MESA) or percutaneous epididymal sperm aspiration (PESA) showed a higher risk of birth defects $[11,18]$. Furthermore, many people over the globe consumed locally grown plants/herbs as nutritional supplements and various therapeutic herbal formulas have been clinically evaluated and approved for fabrication of drugs in developed and developing countries to energize, bolster, and enhance sexual ability eventually to treat various sexual disorders [17, 19-21]. In the outlook of oligospermia, diverse indigenous herbs have been claimed to have sexual arousal effect or even useful for the management of low sperm count [22-27]. However, these herbs proved limited potential for improving the major assessed parameters for semen; in addition less literature has been cited for potential herbal remedy for oligospermia [28-36]. Therefore, there is an immediate need to fabricate a safe and cost-effective therapeutic drug from novel natural sources that devoid of all limitations stated above from modern medicines or/medical techniques and herbal remedies.

Since PHF due to their synergistic effects and multiple biological functions demonstrated the vast advantages over single herbal formulation, therefore, in the present investigation, a PHF was made, based on recommended ayurvedic practicing knowledge/literature citation, which is root of Chlorophytum borivilianum, seeds of Hygrophila spinosa T. Anders, seeds of Mucuna pruriens, seeds of Mimosa pudica, sap of Acacia senegal, root of Astragalus membranaceus, seed coat of Plantago ovate, sap of Bombax ceiba, and root of Eurycoma longifolia and nonherbal constitute called "rock candy". All stated constituents were fabricated into a therapeutic PHF to determine the effects on men infertility. Although, there were several evidences on animal models regarding the sex-stimulating effect of above stated herbs on male fertility and sperm quality and other combination of herbs [28-38]. However no clinical trial has been performed yet to investigate the spermatogenic effect on human. For the reason, a proposed research trial was conducted to determine the synergistic spermatogenic potential of polyherbal formulation on reproductive function of oligospermic male patients.

\section{Material and Methods}

Ethics Statement. This study was approved by the Institutional Review Board on Human Research of University of Lahore Teaching Hospital, Lahore, Pakistan, with reference number (IRB-UOL-FAHS/B00112A). The study was conducted according to the declaration of Helsinki principles. Written informed consent for evaluation and use of their clinical data for scientific purposes was obtained from each patient prior to recruitment.

2.1. Polyherbal Formulation. In the present investigation, PHF was made by combining the root of Chlorophytum borivilianum, seeds of Hygrophila spinosa T. Anders, seeds of Mucuna pruriens, seeds of Mimosa pudica, sap of Acacia senegal, root of Astragalus membranaceus, seed coat of Plantago ovate, sap of Bombax ceiba, root of Eurycoma longifolia, and rocky candy in a ratio of 1:1:1:1:1:1:1:1:1:8 respectively (Table 1), to determine the effect of the prepared formulation on oligospermic patients.

2.2. Preparation of Extract. Samples were thoroughly washed with deionized water to remove any contamination, ground to make crude powder with the help of liquid nitrogen followed by individual ethanol, methanol, acetone, and water extraction. Solvents are selected based on their polarities, from high to low (i.e., water $>$ methanol $>$ ethanol $>$ acetone) as different classes of bioactive compounds possessed different affinities towards the solvents of different polarities.

For the extraction, an aliquot of crude powder $(5 \mathrm{~g}$ ) was accurately weighed into four 250-mL flasks and shaken overnight $(16-20 \mathrm{~h})$ with $50 \mathrm{ml}$ of ethanol, methanol, acetone, and water individually in ratio of 1:10 at room temperature. After the overnight shaking, all samples were centrifuged at $4000 \mathrm{rpm}$ for 10 minutes, followed by the vacuum filtration of supernatant through Whatman paper No. 1 (x2). The residue was then resuspended in another $50 \mathrm{ml}$ of ethanol methanol, acetone, and water, respectively. The process was repeated twice. The combined filtrate was transferred to a preweighted glass plate and the organic solvents were evaporated by placing at hot air oven at $40^{\circ} \mathrm{C}$ for 4 hours after which freeze-drying of filtrate was performed using an Alpha 1-4 LD plus freeze-drying unit (Labconco, Germany). After lyophilization, dry weight of sample was recorded and stored at $-80^{\circ} \mathrm{C}$ for further usage. 
TABLE 1: Composition of polyherbal formulation.

\begin{tabular}{llll}
\hline Scientific Name & Extraction material & Quantity (g/100 g of formulation) & Active Principles \\
\hline $\begin{array}{l}\text { Chlorophytum borivilianum } \\
\text { Hygrophila spinosa T. Anders }\end{array}$ & Roots & 5.88 & Alkaloid, steroids \\
Mucuna Pruriens & Seeds & 5.88 & Terpenoids, phenolics \\
Mimosa pudica & Seeds & 5.88 & Alkaloids \\
Flavonoid, terpenoids, \\
glycosides, alkaloids
\end{tabular}

2.3. Standardization of Polyherbal Formulation (PHF). PHF was standardized as per WHO guidelines. Thin Layer Chromatography (TLC) was executed for three different batches, and the $\mathrm{R}_{\mathrm{f}}$ value was determined for standardization parameters. Quantitative analysis of raw material was also carried out for organoleptic characteristics, physicochemical parameters, and toxicological parameters.

\subsection{Sample Collection and Laboratory Methodology. Semen} sample was collected at morning by patient masturbation into sterile plastic specimen cup in the hospital and all semen values were determined with compliance with World Health Organization (WHO) recommendations at the time of recruitment $[39,40]$. Subjects were instructed to abstain from ejaculation for at least 72 hours prior to producing the semen samples. Samples were left to liquefy for 30 minutes but no longer than one hour prior to performing the semen analysis. To determine semen volume, samples were left to liquefy in an incubator at $37^{\circ} \mathrm{C}, 5 \% \mathrm{CO}_{2}$ for 30 minutes before volume was measured, and sperm concentration and motility parameters were measured by means of computer assisted semen analyzer (CASA) (Hamilton-Thorne Version 10HTMIVOS) using 2 Chamber Leja slides (Leja, The Netherlands). Setting parameters and the definition of measured sperm motion parameters for CASA were established by the manufacturer. A $5 \mu$ l well-mixed, homogeneous sample was applied to each chamber of the Leja slide. Two known concentrations of Accu-bead ${ }^{\circledR}+$ (Hamilton-Thorne, Inc., Beverly, MA, USA) were used each day for quality control and to confirm accuracy of the CASA counting. The Leja slide was placed on the warm CASA stage and analyzed. A manual count of the same sample was also performed and discrepancies $>10 \%$ required a repeat count. On manual count, sperm concentration was obtained by averaging the total number of sperm in both chambers on the Leja slide. At least 100 sperm cells were counted for motility assessment. Reference values from the World Health Organization (WHO) were used to assess sperm concentration and motility [39]. Testosterone, LH and FSH levels of blood serum were assessed using the Immulite automated chemiluminescence immunoassay analyzer (Immulite; Diagnostic Products Corp., Los Angeles, CA, USA) according to manufacturer's instructions, at University of Lahore Teaching Hospital. For IMMULITE 1000 system analytical sensitivity was $0.1 \mathrm{IU} / \mathrm{L}$ for both $\mathrm{LH}$ and FSH. The intra- and interassay CVs were 6.3 and $9.4 \%$ for testosterone, 4.0 and $7.1 \%$ for $\mathrm{LH}$, and 4.2 and $8 \%$ for FSH.

2.5. Subjects. This double blind, two-armed, placebocontrolled, randomized, comparable group trial with 1:1 arbitrary allocation was prospectively conducted at University of Lahore Teaching Hospital, Punjab, Pakistan, between September 2016 and December 2016. Out of ninety-one (91) patients, thirty-six (36) oligospermic patients were selected for the current clinical trial after predefined scrutiny. All participants in both groups were neither alcoholic nor user for any tobacco product. All oligospermic patients between the ages of 22 and 40 years were registered after acquiring the written approval from them; they also had a background of accustomed sexual intercourse over 12 months with a sexually normal female partner. All oligospermic patients exhibited the sperm count between the range of 0.0 to 15.0 million $/ \mathrm{mL}$. Subject with a total sperm count below the 0.0 million $/ \mathrm{mL}$ or greater than $15 \mathrm{million} / \mathrm{mL}$ were not considered. Subject having cardiovascular diseases (CD), erectile dysfunction (ED), varicocele, testicular hypertrophy, uncontrolled diabetes mellitus (DM), obesity, precise hepatic or renal disorder, and cerebrovascular problems or with previous history of partly obstructive oligospermia, pelvic fractures prosthetic surgery on the penis, and cryptorchidism were also debarred from the current clinical trial. Oligospermic patients with known allergic reaction to any constitute of polyherbal extract were also not included.

2.6. Randomization and Treatments. The subjects for trial were randomized to PHF treated group $(n=23)$ and placebo group $(n=13)$. The oligospermic patients in PHF treated group were administered 3 capsules per day (containing $250 \mathrm{mg}$ of high spectrum of PHF-extract) orally for a period of 3 months (90 days) on the other hand, capsules containing 
$250 \mathrm{mg}$ of corresponding placebo were given in the same way to the placebo group.

The constituents used for formulation of polyherbal extract for the current trial had been extracted with an exclusive processing technology for developing a great phytopharmaceutical feature that amplified the action of PHF-extract manifolds, ultimately providing pan-therapeutic effects.

2.7. Trial Visit and Assessments. Throughout the treatment phase of 90 days ( 3 months), the subjects were mandatory to present themselves at the trial hub at specified intervals: Visit 01 on Day 60 and Visit 02 on Day 90 (3 months). Complete physical examination was conducted at baseline (Day 0), height and weights were measured by physician balance beam scale with height rod, while respiratory rate blood pressure and body temperature were assessed by Vital Signs Monitoring System (VSMS) and semen analysis was done at predefined time intervals likewise at Day 0, then after 60 days and 90 days. Semen analyses were performed according to World Health Organization 2010 criteria [39, 40]. Serum testosterone, luteinizing hormone ( $\mathrm{LH})$, and serum folliclestimulating hormone (FSH) levels were evaluated on Day 0 (baseline) and then after Day 90. The principal (primary) usefulness result was the advancement in the major semen parameters and serum hormone level from baseline (Day 0) after 90 days of treatment.

2.8. Statistical Methods. In the current study, placebo group and PHF treated groups were evaluated for change in the semen parameters from the baseline (Day 0) by using oneway ANOVA (with treatment as a factor). The acquired outcome was taken as insignificant if the $P$ value exceeded to 0.05 .

\section{Results}

3.1. Composition of Polyherbal Formulation. Recently, polyherbal formulations (PHFs) are gaining importance all over the globe due to their dynamic medicinal and therapeutic claims and demonstrated vast advantages over single herbal formulations (SHFs), in the outlook of aforementioned claims; we have prepared a novel polyherbal formulation (PHF) to recover the male patients from oligospermia, and its composition is described in Table 1.

3.2. Standardization of Polyherbal Formulation (PHF). TLC was run for the ethanolic extract in the solvent system ethanol/acetone/water at 17:2:1 ratio and plates were visualized directly after drying under UV at $254 \mathrm{~nm}$ and $366 \mathrm{~nm}$ in UV TLC viewer and then sprayed the TLC plates with a solution of $37 \%$ formaldehyde in conc. sulfuric acid (1:10) (for alkaloids), 1\% ethanolic solution of aluminum chloride (for flavonoids), and p-anisaldehyde-sulfuric acid reagent (for phenols, sugars, steroids, and terpenoids) according to standard protocols. There were six spots evidently observed in all three batches and $R_{f}$ values were calculated as 0.82 , $0.77,0.7,0.67,0.53$, and 0.31 in each replicate. All other factors likewise organoleptic characteristics, physicochemical parameters, and toxicological parameters were also compliance with WHO recommendations and elaborated in Table 2.

3.3. Demographic Parameters and Participants Distribution. Ninety-one infertile males $(n=91)$ were taken into consideration for a proposed research study. Thirty-six $(n=36)$ were finally selected for clinical trial. This trial demonstrated the statistics of those 36 oligospermic patients that were double blind randomly distributed into two groups, i.e., the high-concentration PHF treated group $(n=23)$ and placebo group $(n=13)$. The two groups were comparable in terms of demographic parameters and demonstrated all vitals in optimum range (Table 3 ).

3.4. Semen Parameters. PHF treated group showed a highly significant $(P \leq 0.001)$ enhancement in sperm concentration after 90 days ( 3 months) of treatment as compared to the baseline value on Day 0 of the current trial (Figure 1(a)). The boost in sperm concentration was from $10.04 \pm 3.21 \times 10^{6} / \mathrm{mL}$ to $35.82 \pm 5.11 \times 10^{6} / \mathrm{mL}$, corresponding to a percentage increase of $256 \%$. A significant augmentation was also recorded in the semen volume (from $1.7 \pm 0.14 \mathrm{~mL}$ to $4.32 \pm 0.38 \mathrm{~mL} ; P \leq 0.001$ ) (Figure 1(b)) and sperm motility (from $15.43 \pm 2.40 \%$ to $48.65 \pm 5.10 \% ; P \leq 0.001$ ) (Figure 1(c)) on Day 90 (3 months) in contrast to the baseline value on Day 0 . These corresponded to increases of $154 \%$ and $215 \%$, respectively.

3.5. Serum Hormone Levels. Moreover, serum hormones levels were also improved appreciably likewise testosterone level had been boosted up by $112 \%$ (from $3.65 \pm 0.71 \mathrm{ng} / \mathrm{mL}$ to $7.76 \pm 0.85 \mathrm{ng} / \mathrm{mL} ; P \leq 0.001$ ) (Figure $2(\mathrm{a})$ ) and $\mathrm{LH}$ by $69 \%$ (from $3.38 \pm 0.91 \mathrm{mIU} / \mathrm{mL}$ to $5.73 \pm 1.32 \mathrm{mIU} / \mathrm{mL} ; P$ $\leq 0.05$ ) (Figure 2(b)) while FSH level had been declined by $37 \%$ (from $26.45 \pm 7.90 \mathrm{mIU} / \mathrm{mL}$ to $16.65 \pm 4.21 \mathrm{mIU} / \mathrm{mL} ; P$ $\leq 0.05$ ) (Figure $2(\mathrm{c})$ ) with subsequent treatment with PHFextract, in contrast to the baseline (Day 0) values of these parameters.

\section{Discussion}

Infertility is defined as not being able to achieve pregnancy (conceive) after one year of isolated sex with the same partner. One year is lower reference limit (LRL) time for conceive decided by the World Health Organization [3, 41, 42]. Among infertility, male infertility accounts for about $50 \%$ of human infertility and considered to be less complicated than female infertility $[3,4,43]$. Several investigations had earlier confirmed that undermined semen production and sperm quality are amid the imperative causative factors for male infertility $[5,6]$. In addition to different physical defects, oligospermia is the widespread cause of male infertility in more than $90 \%$ of the cases. Out of these, about 30 to $40 \%$ causes are unexplained, and in the rest of the cases critical illness, genetic abnormalities, malnutrition, pollution, side effects of some medicines, hormones, and chemicals play a key role in infertility $[1,2]$. In the present investigation, we formulated a polyherbal formulation (PHF) based on previous therapeutic scientific endeavors for the critical 
TABLE 2: Standardization of polyherbal formulation (capsule).

\begin{tabular}{|c|c|}
\hline Name of Test & Observations \\
\hline \multicolumn{2}{|l|}{ Organoleptic characteristics } \\
\hline Description & Grayish brown colored Powder filled in blue cap/red body, 0 sized capsule \\
\hline Color & Grayish brown \\
\hline Odor & Characteristics \\
\hline Taste & Slightly Sweet \\
\hline \multicolumn{2}{|l|}{ Physicochemical Parameters } \\
\hline $\mathrm{pH}$ & 7.6 \\
\hline Moisture Contents (\%) & 1.04 \\
\hline Average Weight (mg) & 251 \\
\hline Weight Variations (Mean \pm SEM) & $249-253$ \\
\hline Loss on drying $(\%)$ & 1.2 \\
\hline Total ash (\%) & 6.54 \\
\hline Water soluble ash (\%) & 3.85 \\
\hline Acid-insoluble ash (\%) & 1.4 \\
\hline Ethanol Soluble Extractive value (\%) & 19.45 \\
\hline Methanol Extractive value (\%) & 15.02 \\
\hline Water Soluble Extractive value (\%) & 13.34 \\
\hline \multicolumn{2}{|l|}{ Toxicological Parameters } \\
\hline Arsenic (ppm) & $1.0 \mathrm{ppm}$ \\
\hline Lead (ppm) & $0.5 \mathrm{ppm}$ \\
\hline Total microbial count NMT $1000 \mathrm{cfu} / \mathrm{g}$ & $105 \mathrm{cfu} / \mathrm{g}$ \\
\hline Yeasts and molds & Nil \\
\hline Presence of E. coli & Absent \\
\hline Presence of Streptococcus & Absent \\
\hline Presence of Salmonella & Absent \\
\hline Presence of Pseudomonas & Absent \\
\hline
\end{tabular}

TABLE 3: Demography and baseline data of the study subjects.

\begin{tabular}{lll}
\hline Parameters & Placebo & PHF- Treated \\
\hline Age $(\mathrm{yr})$ & $28.53 \pm 3.83$ & $29.78 \pm 4.75$ \\
Height $(\mathrm{cm})$ & $165.46 \pm 9.05$ & $165.47 \pm 12.30$ \\
Weight $(\mathrm{Kg})$ & $72.46 \pm 6.04$ & $75.91 \pm 7.10$ \\
Pulse (per min) & $79.53 \pm 6.56$ & $81.39 \pm 6.06$ \\
Respiratory Rate (per minute) & $14.6 \pm 1.45$ & $14.34 \pm 1.61$ \\
Systolic Blood Pressure (mm Hg) & $123.66 \pm 7.06$ & $125.82 \pm 4.09$ \\
Diastolic Blood Pressure (mm Hg) & $84 \pm 3.46$ & $84.47 \pm 2.59$ \\
Body Temperature $\left({ }^{\circ} \mathrm{F}\right)$ & $98.54 \pm 0.30$ & $98.60 \pm 0.41$ \\
\hline
\end{tabular}

oligospermic patients to recover their sexual potency. Among the key ingredients, we selected Chlorophytum borivilianum due to its aphrodisiac action, used to cure impotency and sterility and enhance male sexual potency ultimately for the treatment of certain forms of sexual inadequacies, such as premature ejaculation and oligospermia [28, 44]. Similar kinds of findings were also reported earlier, as discussed below in detail, by various investigators, and highlighted their positive outcomes on the oligospermia and different semen parameters using in vitro/in vivo model systems for Hygrophila spinosa T. Anders [29], Mucuna pruriens [30], Mimosa pudica [31], Acacia Senegal [32], Astragalus membranaceus [33], Plantago ovate [34], Bombax ceiba [35], and Eurycoma longifolia [36].

To the best of our acquaintance, this is the first demonstration to explore the synergistic efficacy of our prepared PHF on human clinical trial, which leads to the improvement in the major semen parameters of oligospermic males compared with placebo group. In this clinical study, we noticed highly significant improvements in major assessed parameters of semen such as sperm concentration, semen volume, and sperm motility in PHF treated group compared to placebo group. Serum hormones like testosterone, LH, and FSH were also markedly improved. Serum testosterone, 


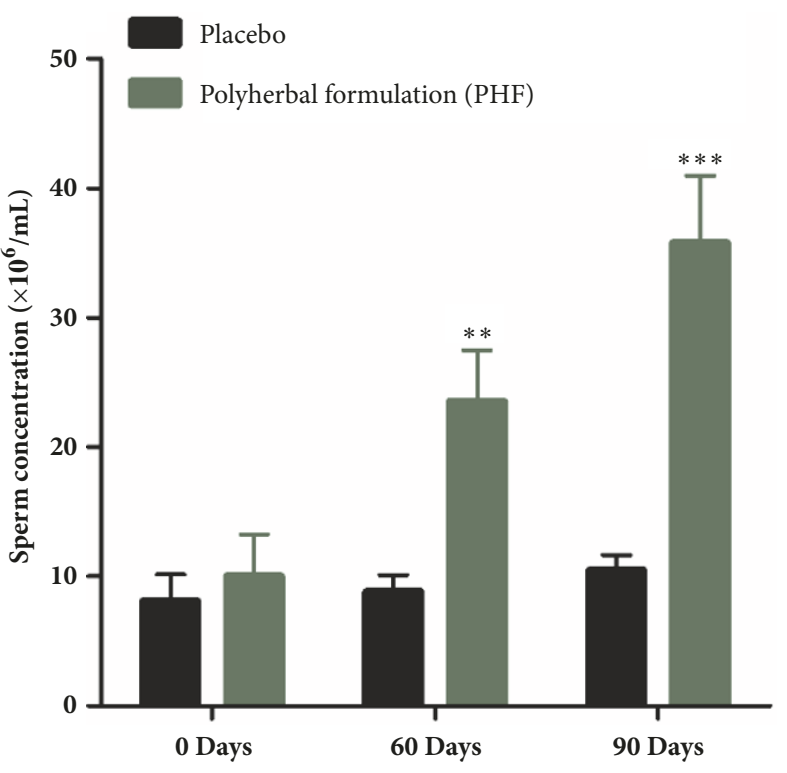

(a)

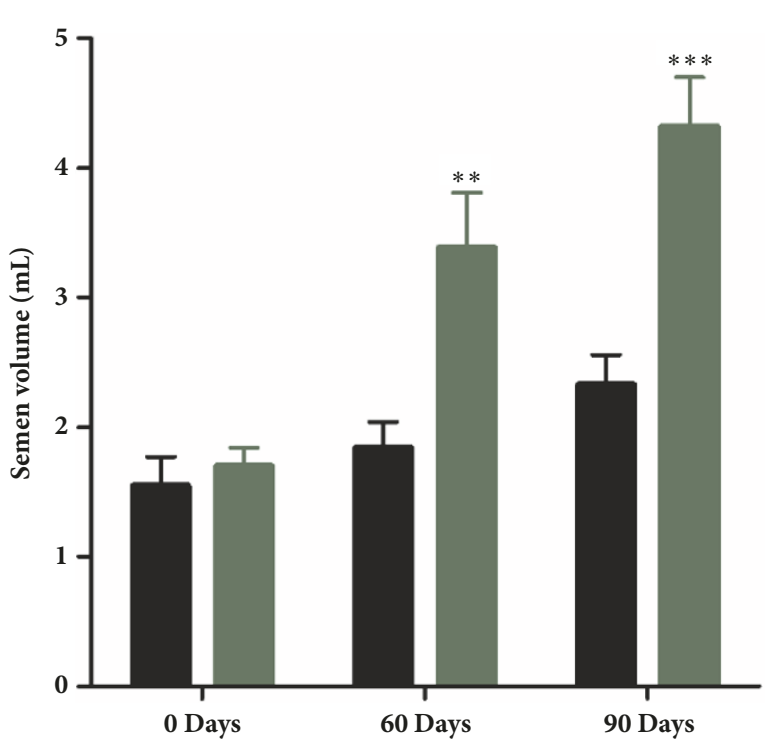

(b)

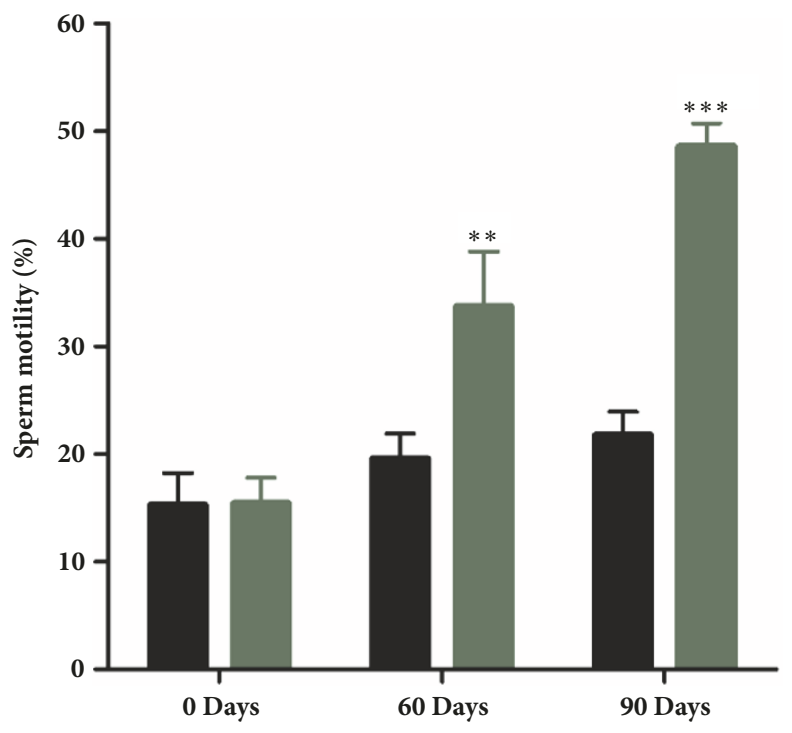

(c)

Figure 1: (a) Sperm concentration $\left(\times 10^{6} / \mathrm{mL}\right)$, (b) semen volume $(\mathrm{mL})$, and (c) sperm motility (\%) in the full-spectrum PHF treated group and placebo group including oligospermic males. $* * P \leq 0.001$ as compared to baseline values on Day 0 of the study duration of 3 months. Values are expressed as mean $\pm \mathrm{SD}$.

$\mathrm{LH}$, and FSH are the hormonal markers of androgenicity [45]. An elevated FSH level is generally designated to severe seminiferous epithelium damage [11] and it is inversely correlated with sperm concentration motility and morphology [46]. Among these biomarkers the serum testosterone and LH levels were increased up to $112 \%$ and $69 \%$, respectively, and conversely FSH level was declined up to $37 \%$ in PHF treated group as compared to placebo group. The boost in serum testosterone and LH level and drop in FSH level may be predominantly attributed to herb Mucuna pruriens as it was documented that Mucuna pruriens increased epididymal alkaline phosphatase activity, protein level in epididymis, serum testosterone, LH, dopamine, adrenaline, and noradrenaline levels in infertile men and reduced the levels of FSH and PRL (Prolactin) [30, 47] and ultimately enhanced spermatogenesis due to the presence of high contents of alkaloids [48, 49]. Many authors also cited the antioxidant, antitumor, and anti-inflammatory impending of alkaloids [44]. The escalation in serum testosterone is also endorsed to another herb called Eurycoma longifolia which was previously reported for its ability to activate $17 \alpha$-hydroxylase/17, 20 lyase (CYP17) enzyme that would augment testosterone level [50]. In addition, another clinical study on human, also declared that Eurycoma longifolia supplementation increased 


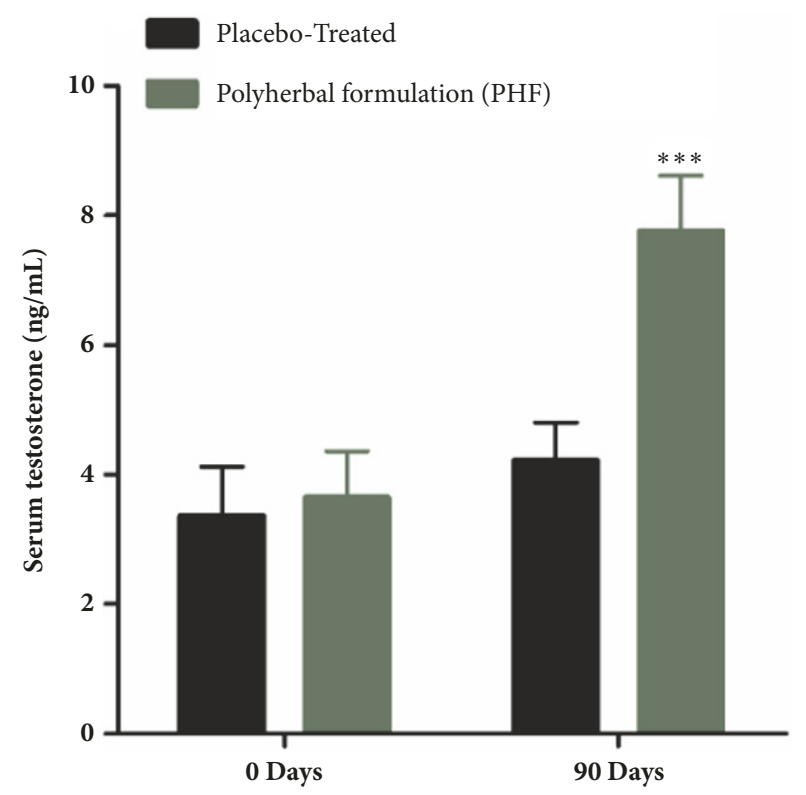

(a)

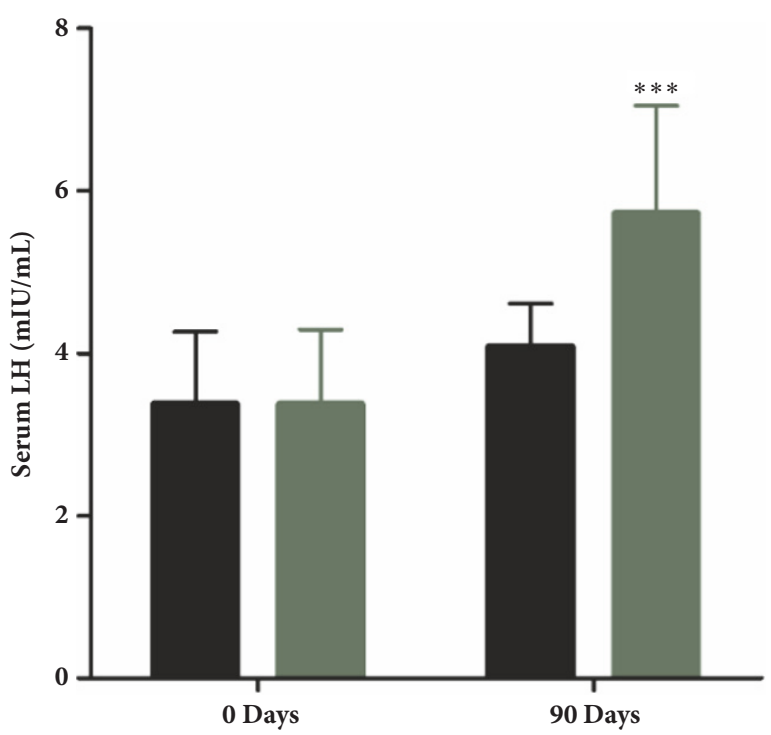

(b)

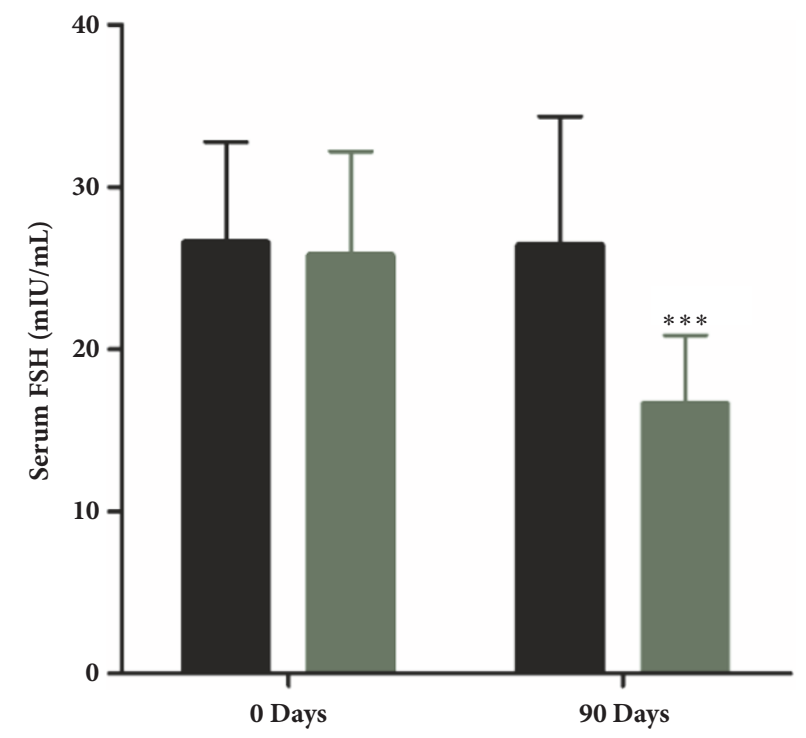

(c)

Figure 2: (a) Serum testosterone levels $(\mathrm{ng} / \mathrm{mL})$, (b) serum LH $(\mathrm{mL} I U / \mathrm{mL})$, and (c) serum FSH (mL IU/mL) in the full-spectrum PHF treated group and placebo group including oligospermic males. $* * P \leq 0.001$ as compared to baseline values on Day 0 of the study duration of 3 months. Values are expressed as mean \pm SD.

the level of testosterone [51]. An increase in testosterone level has been associated with increase of sexual desire, penile tumescence, and rigidity, as well as the accessory muscles which help to strengthen and provide additional sexual activity [52-54]. Research with various animal and human models pointed out that there is a strong correlation between sexual behavior and brain neurotransmitters like dopamine, 5- hydroxytryptamine (5-HT), serotonin, and nitrergic neurons [55]. The motor switch of ejaculation in animals is also governed by serotonin and its receptors [56]. Testosterone may also expedite male sexual performance by aggregative dopamine discharge in the medial preoptic area and potentiating nitrergic neurotransmission in brain, which ensued in stimulation of hypothalamic-pituitary-gonadal axis $[57,58]$. Hike in the testicular weight symbolizes the number as well as sperms motility [59]. Increased serum testosterone and LH levels and decreased FSH level after administration of PHF could thus be considered as one of the imparting factors accountable for the overall improved sexual potential against oligospermia in all PHF treated groups compared to placebo group.

The credit for a boost in endogenous testosterone levels and enhancing male sexual behavior goes to possible bioactive agents present in our novel formulation. 
These bioactive components may be steroids, antioxidants, saponins, peptides, organic acids, etc. and our future research will be comprised of characterizing and elucidating these novel bioactive compounds. The stated mechanisms of these agents include steroids by rising androgen production [60, 61], flavonoids by augmenting testosterone synthesis or by inhibiting its metabolic degradation [62, 63], alkaloids [52] by dilating the blood vessels in the sexual organs [64], and saponins by activating gonadal tissues and central nervous system via NO-dependent mechanism [65]. Thus, the improvements in sexual function established in the current study might be due to the existence of such compounds in our prepared PHF.

Sperm motility is also a very important factor for the proper fertilization and conceivability. Sperm motility was also significantly increased up to $215 \%$ as compared to placebo group in our study. This increase is mainly accredited by Astragalus membranaceus as at the dose of $10 \mathrm{mg} / \mathrm{ml}$; the sperm motility in semen has increased up to $146.6+/-22.6 \%$ of control and for washed sperm increased up to $138.2+/$ $13.8 \%$ of control [33].

Nevertheless, sperm count and semen volume were also significantly increased up to $256 \%$ and $154 \%$, respectively, in PHF treated as compared to placebo group. It may due to individual or synergistic activity of other PHF ingredients which had demonstrated previously proven spermatogenetic potential and aphrodisiac activity for alleviating oligospermia. Likewise, seeds of Hygrophila spinosa T. Anderson have spermatogenic effect which may be owing to the hormonal and neurohumoral changes, which play a vital role in the sexual behavior and fertility disorders [29]. Mimosa pudica seeds extract possesses protective, therapeutic, and restorative capacity on the histoarchitecture of hypothalamicpituitary-testicular axis components [31]. Acacia Senegal enhanced the semen quality and could protect testis via improvement of antioxidant capacity; it may be useful to overcome the diabetic fertility complications [32]. Plantago ovate seed used in considerable quantity in PHF ultimately facilitated recovering the oligospermia and subsidized the male infertility [34]. Bombax ceiba root extract enhanced the spermatogenesis, sexual behavior, and erectile function [35]. Eurycoma longifolia Jack plant may potentially be suitable for the management of oligospermia and male infertility $[36,66-$ 68]. Our finding is also more significant compared with previously designed PHF which increased the sperm count maximum up to $39 \%$ to $40.5 \%$ in different animal models $[37,38]$.

It can be accomplished from above discussion that our currently designed PHF demonstrated more significant results compared with previous work on various animal and human models for individual component of current PHF or combination of two or more of other PHFs. In addition, aforementioned PHF also revealed diverse advantages over western medicine/medical techniques, i.e., higher expenditure associated with assisted reproductive technology (ART), ART affects patient's physical and emotional wellbeing may contribute psychological distress, caused birth defects, and proved less live birth rate [11, 1736].

\section{Conclusion}

PHF could improve the quantity and quality of semen in a statistically significant manner in oligospermia male adults between the ages of 22 to 40 years, in comparison to the placebo, when used for 90 days, at $750 \mathrm{mg} / \mathrm{d}$ in three doses. PHF does also improve the serum testosterone; $\mathrm{LH}$; and FSH level in a majority of PHF treated males, in comparison to the placebo. The results suggested that the prepared PHF may be a new auspicious novel therapeutic amalgamation, which can be used to improve the spermatogenic potential of many oligospermic infertile men. This spermatogenic property may be due to possible synergistic action of selected herbs' parts used in the preparation of PHF. However, further investigations are warranted to confirm and elucidate the effect of PHF on semen parameters.

\section{Data Availability}

The data used to support the findings of this study are available from the corresponding author upon request.

\section{Authors' Contributions}

Yuanda Song supervised this work. Syed Ammar Hussain and Ahsan Hameed performed all the experimental work. Syed Ammar Hussain wrote this manuscript. Furqan Nasir carried out the clinical tests. Yang Wu analyzed the data and helped in the statistical analysis. Hafiz Ansar Rasul Suleria edited and reviewed the whole manuscript and provided suggestions to main authors about overall research plan. Syed Ammar Hussain and Ahsan Hameed contributed equally to this work. All authors read and approved the final manuscript.

\section{Conflicts of Interest}

No conflicts of interest were reported by authors.

\section{Acknowledgments}

The authors are indebted to herbal medicine practitioner Mr. Syed Ijaz Hussain for kindly providing the specimen herbal plants and helping in formulating the PHF. They are also thankful to Dr. Furqan Nasir for proving space and assistance in carrying out the clinical tests. This work is supported by National Natural Science Foundation, China (Grant no. 31670064), and TaiShan Industrial Experts Programme.

\section{Supplementary Materials}

The graphical presentation of whole study has been provided in supplementary material. (Supplementary Materials)

\section{References}

[1] C. Haslett, E. R. Chilvers, N. A. Boon, and N. A. Colledge, Davidsons Principles and Practice of Medicine, Churchill Livingstone, Edinburgh, UK, 2002. 
[2] T. K. Biswas, S. Pandit, S. Mondal et al., "Clinical evaluation of spermatogenic activity of processed Shilajit in oligospermia," Andrologia, vol. 42, no. 1, pp. 48-56, 2010.

[3] T. G. Cooper, E. Noonan, S. von Eckardstein et al., "World Health Organization reference values for human semen characteristics," Human Reproduction Update, vol. 16, no. 3, pp. 231245, 2009.

[4] "Report on optimal evaluation of the infertile male," Fertility and Sterility, vol. 82, pp. 123-130, 2004.

[5] V. M. Brugh and L. I. Lipshultz, "Male factor infertility: evaluation and management," Medical Clinics of North America, vol. 88, no. 2, pp. 367-385, 2004.

[6] R. K. Mishra, H. P. Verma, N. Singh, and S. K. Singh, "Male infertility: lifestyle and oriental remedies," Journal of Scientific Research, vol. 56, pp. 93-101, 2012.

[7] R. B. Gwatkin, "Male Infertility: The Interpretation of The Diagnostic Assessment," in The Year Book of Infertility, Mishell D. R., R. A. Lobo, and C. A. Paulsen, Eds., Book Medical Publishers, Chicago, Ill, USA, 1989.

[8] Vital and Health Statistics, no. 26, CDC, 2002, http://www.cdc .gov.

[9] D. F. Yao and J. N. Mills, "Male infertility: Lifestyle factors and holistic, complementary, and alternative therapies," Asian Journal of Andrology, vol. 18, no. 3, pp. 410-418, 2016.

[10] S. Bisht, M. Faiq, M. Tolahunase, and R. Dada, "Oxidative stress and male infertility," Nature Reviews Urology, vol. 14, no. 8, pp. 470-485, 2017.

[11] O. Khorram, P. Patrizio, C. Wang, and R. Swerdloff, "Reproductive Technologies for Male Infertility," The Journal of Clinical Endocrinology \& Metabolism, vol. 86, no. 6, pp. 2373-2379, 2001.

[12] M. Punab, O. Poolamets, P. Paju et al., "Causes of male infertility: A 9-year prospective monocentre study on 1737 patients with reduced total sperm counts," Human Reproduction, vol. 32, no. 1, pp. 18-31, 2017.

[13] E. Ashamu, E. Salawu, O. Oyewo, A. Alhassan, O. Alamu, and A. Adegoke, "Efficacy of vitamin $\mathrm{C}$ and ethanolic extract of Sesamum indicum in promoting fertility in male Wistar rats," Journal of Human Reproductive Sciences, vol. 3, no. 1, pp. 11-14, 2010.

[14] P. Berger, M. Gruschwitz, G. Spoettl et al., "Human chorionic gonadotropin (hCG) in the male reproductive tract," Molecular and Cellular Endocrinology, vol. 260-262, pp. 190-196, 2007.

[15] C. Foresta, R. Selice, A. Garolla, and A. Ferlin, "Folliclestimulating hormone treatment of male infertility," Current Opinion in Urology, vol. 18, no. 6, pp. 602-607, 2008.

[16] M. Walschaerts, L. Bujan, J. Parinaud, R. Mieusset, and P. Thonneau, "Treatment discontinuation in couples consulting for male infertility after failing to conceive," Fertility and Sterility, vol. 99, no. 5, pp. 1319-1323, 2013.

[17] D. L. Rowland and W. Tai, "A review of plant-derived and herbal approaches to the treatment of sexual dysfunctions," Journal of Sex \& Marital Therapy, vol. 29, no. 3, pp. 185-205, 2003.

[18] J. Wen, J. Jiang, C. Ding et al., "Birth defects in children conceived by in vitro fertilization and intracytoplasmic sperm injection: A meta-analysis," Fertility and Sterility, vol. 97, no. 6, pp. 1331-e4, 2012.

[19] S. K. Kwon, H. Y. Lee, M. J. Kang, and D. I. Kim, "Effect of herbmedicine on seminal parameters in male infertility patients," The Journal of Korean Obstetrics and Gynecology, vol. 18, pp. 184-191, 2005.
[20] Z. G. Sun, F. Lian, K. P. Jiang, J. W. Zhang, F. M. Ma, N. Zhang et al., "Shengjing prescription improves semen parameters of oligoasthenozoospermia patientsefficacy and mechanism," National journal of andrology, vol. 18, pp. 764-767, 2012.

[21] H. G. Tempest, S. T. Homa, X. Zhai, and D. K. Griffin, "Significant reduction of sperm disomy in six men: effect of traditional Chinese medicine?" Asian Journal of Andrology, vol. 7, no. 4, pp. 419-425, 2005.

[22] F. L. Gold, N. Lewin, N. Flomenbaum, and M. A. Hawland, "The pernicious panacea: herbal medicine," Hospital Physician, vol. 18, pp. 64-87, 1982.

[23] A. G. Vulto and P. A. G. M. Smet, "Drug used in non-orthodox medicine," in Meyler's Side Effects of Drugs, vol. 10, pp. 99-105, Elsevier, Amsterdam, Netherlands, 11th edition, 1988.

[24] J. F. Smith, M. L. Eisenberg, S. G. Millstein et al., "The use of complementary and alternative fertility treatment in couples seeking fertility care: Data from a prospective cohort in the United States," Fertility and Sterility, vol. 93, no. 7, pp. 2169-2174, 2010.

[25] S. K. Bardaweel, M. Shehadeh, G. A. R. Y. Suaifan, and M.-V. Z. Kilani, "Complementary and alternative medicine utilization by a sample of infertile couples in Jordan for infertility treatment: Clinics-based survey," BMC Complementary and Alternative Medicine, vol. 13, article no. 35, 2013.

[26] M. Stankiewicz, C. Smith, H. Alvino, and R. Norman, "The use of complementary medicine and therapies by patients attending a reproductive medicine unit in South Australia: A prospective survey," Australian and New Zealand Journal of Obstetrics and Gynaecology, vol. 47, no. 2, pp. 145-149, 2007.

[27] M. T. Sultan, M. S. Buttxs, M. M. Qayyum, and H. A. Suleria, "Immunity: Plants as Effective Mediators," Critical Reviews in Food Science and Nutrition, vol. 54, no. 10, pp. 1298-1308, 2014.

[28] R. Kenjale, R. Shah, and S. Sathaye, "Effects of Chlorophytum borivilianum on sexual behaviour and sperm count in male rats," Phytotherapy Research, vol. 22, no. 6, pp. 796-801, 2008.

[29] A. K. Sahoo and B. Gandhare, "Effect of Hygrophila spinosa T. on Reproductive Function of Male Albino Rats," Journal of Complementary and Integrative Medicine, vol. 7, no. 1, 2010.

[30] K. K. Shukla, A. A. Mahdi, M. K. Ahmad, S. N. Shankhwar, S. Rajender, and S. P. Jaiswar, "Mucuna pruriens improves male fertility by its action on the hypothalamus-pituitary-gonadal axis," Fertility and Sterility, vol. 92, no. 6, pp. 1934-1940, 2009.

[31] E. E. Ekpenyong, E. L. Anderson, T. A. Abdullateef et al., "The impact of Mimosa pudica on the Histoarchitecture of hypothalamic-pituitary-testicular axis in cadmium treated rats," World Journal of Pharmacy And Pharmaceutical Sciences, vol. 4, pp. 169-179, 2015.

[32] J. S. Fedail, A. A. Ahmed, H. H. Musa, E. Ismail, A. Z. Sifaldin, and T. H. Musa, "Gum arabic improves semen quality and oxidative stress capacity in alloxan induced diabetes rats," Asian Pacific Journal of Reproduction, vol. 5, no. 5, pp. 434-441, 2016.

[33] C. Y. Hong, J. Ku, and P. Wu, "Astragalus membranaceus stimulates human sperm motility in vitro," American Journal of Chinese Medicine, vol. 20, pp. 289-294, 1992.

[34] P. Parthiban, K. Kanagavalli et al., "Spermatogenetic activity of Isappukol chooranam - Sidha medicine against TCDD induced oligospermic Rats," International Journal of Pharmacy and Biological Sciences, vol. 2, pp. 164-179, 2013.

[35] C. Bhargava, M. Thakur, and S. K. Yadav, "Effect of Bombax ceiba L. on spermatogenesis, sexual behaviour and erectile function in male rats," Andrologia, vol. 44, no. 1, pp. 474-478, 2012. 
[36] K. L. Chan, B. S. Low, C. H. Teh, and P. K. Das, "The effect of Eurycoma longifolia on sperm quality of male rats," Natural product communications, vol. 4, pp. 1331-1336, 2009.

[37] H. B. Sahoo, S. Nandy, A. K. Senapati, S. P. Sarangi, and S. K. Sahoo, "Aphrodisiac activity of polyherbal formulation in experimental models on male rats," Pharmacognosy Research, vol. 6, no. 2, pp. 120-126, 2014.

[38] R. Dhumal, T. Vijaykumar, V. Dighe et al., "Efficacy and safety of a herbo-mineral ayurvedic formulation 'afrodet Plus ${ }^{\circledR}$ ' in male rats," Journal of Ayurveda and Integrative Medicine, vol. 4, no. 3, pp. 158-164, 2013.

[39] V. Prasad, U. Schwerdtfeger, F. El-Awa, D. Bettcher, and V. da Costa e Silva, "Closing the door on illicit tobacco trade, opens the way to better tobacco control," Eastern Mediterranean Health Journal, vol. 21, no. 6, pp. 379-380, 2015.

[40] World Health Organization, WHO Laboratory Manual For the Examination and Processing of Human Semen, World Health Organization, Geneva, Swizerland, 5th edition, 2010.

[41] C. D. Meletis and J. Barker, "Natural Ways to Enhance Male Fertility," Alternative and Complementary Therapies, vol. 10, no. 1, pp. 22-27, 2004.

[42] R. S. Makar and T. L. Toth, "The Evaluation of Infertility," Pathology Patterns Reviews, vol. 117, no. suppl_1, pp. S95-S103, 2002.

[43] S. Norris, "Ottawa: Parliamentary Information and Research Service, Library of Parliament," in Reproductive infertility: Prevalence, causes, trends and treatments, PRB-00-32E, 2001, http://www.parl.gc.ca/Content/LOP/researchpublications/ prb0035-e.pdf.

[44] M. Thakur, N. S. Chauhan, S. Bhargava, and V. K. Dixit, "A comparative study on aphrodisiac activity of some ayurvedic herbs in male albino rats," Archives of Sexual Behavior, vol. 38, no. 6, pp. 1009-1015, 2009.

[45] M. T. Yakubu, M. A. Akanji, A. T. Oladiji, and A. A. Adesokan, "Androgenic potentials of aqueous extract of Massularia acuminata (G. Don) Bullock ex Hoyl. stem in male Wistar rats," Journal of Ethnopharmacology, vol. 118, no. 3, pp. 508-513, 2008.

[46] J. D. Meeker, L. Godfrey-Bailey, and R. Hauser, "Relationships between serum hormone levels and semen quality among men from an infertility clinic," Journal of Andrology, vol. 28, no. 3, pp. 397-406, 2007.

[47] K. Muthu and P. Krishnamoorthy, "Evaluation of androgenic activity of Mucuna pruriens in male rats," African Journal of Biotechnology, vol. 10, no. 66, pp. 15017-15019, 2011.

[48] K. M. Y. Amin, M. N. Khan, S. Zillur-Rehman, and N. A. Khan, "Sexual function improving effect of Mucuna pruriens in sexually normal male rats," Fitoterapia, vol. 67, no. 1, pp. 53-58, 1996.

[49] A. K. V. Kumar and K. K. Srinivasan, "Aphrodisiac activity of the seeds of Mucuna pruriens," Indian Drugs, vol. 31, no. 7, pp. 321-327, 1994.

[50] M. I. B. M. Tambi, "Nutrients and Botanicals for optimizing men's health. Examining the evidence for Eurycoma longifolia Jack, the Malaysian ginsing in men's health," Journal of Andrology, vol. 11, pp. 37-38, 2009.

[51] M. I. Tambi, M. K. Imran, and R. R. Henkel, "Standardised water-soluble extract of Eurycoma longifolia, Tongkat ali, as testosterone booster for managing men with late-onset hypogonadism?" Andrologia, vol. 44, pp. 226-230, 2012.

[52] J. F. Cao, P. Y. Zhang, C. W. Xu, T. T. Huang, Y. G. Bai, S. K. Chen et al., "Effect of aqueous extract of Arctiumlappa L. (burdock) roots on the sexual behavior of male rats," BMC Complementary and Alternative Medicine, vol. 12, pp. 1-8, 2012.

[53] A. Aversa and A. Fabbri, "New oral agents for erectile dysfunction: What is changing in our practice?" Asian Journal of Andrology, vol. 3, pp. 175-179, 2001.

[54] K. Gauthaman, P. G. Adaikan, and R. N. V. Prasad, "Aphrodisiac properties of Tribulus Terrestris extract (Protodioscin) in normal and castrated rats," Life Sciences, vol. 71, no. 12, pp. 13851396, 2002.

[55] S. Ramachandran, Y. Sridhar, S. K. G. Sam et al., "Aphroidisiac activity of Butea frondosa Koen. ex Roxb. extract in male rats," Phytomedicine, vol. 11, no. 2-3, pp. 165-168, 2004.

[56] F. Giuliano and P. Clément, "Physiology of Ejaculation: Emphasis on Serotonergic Control," European Urology, vol. 48, no. 3, pp. 408-417, 2005.

[57] N. S. Chauhan and V. K. Dixit, "Effects of Bryonia laciniosa seeds on sexual behaviour of male rats," International Journal of Impotence Research, vol. 22, no. 3, pp. 190-195, 2010.

[58] S. K. Putnam, J. Du, S. Sato, and E. M. Hull, "Testosterone Restoration of Copulatory Behavior Correlates with Medial Preoptic Dopamine Release in Castrated Male Rats," Hormones and Behavior, vol. 39, no. 3, pp. 216-224, 2001.

[59] G. F. Gonzales, A. Ruiz, C. Gonzales, L. Villegas, and A. Cordova, "Effect of Lepidium meyenii (maca) roots on spermatogenesis of male rats," Asian Journal of Andrology, vol. 3, no. 3, pp. 231-233, 2001.

[60] H. A. R. Suleria, M. S. Butt, F. M. Anjum, F. Saeed, and N. Khalid, "Onion: Nature Protection Against Physiological Threats," Critical Reviews in Food Science and Nutrition, vol. 55, no. 1, pp. 50-66, 2015.

[61] A. Hameed, S. A. Hussain, J. Yang et al., "Antioxidants Potential of the Filamentous Fungi (Mucor circinelloides)," Nutrients, vol. 9, no. 10, p. 1101, 2017.

[62] R. Perveen, H. A. R. Suleria, F. M. Anjum, M. S. Butt, I. Pasha, and S. Ahmad, "Tomato (Solanum lycopersicum) Carotenoids and Lycopenes Chemistry; Metabolism, Absorption, Nutrition, and Allied Health Claims-A Comprehensive Review," Critical Reviews in Food Science and Nutrition, vol. 55, no. 7, pp. 919-929, 2015.

[63] N. J. Yang, K. Kaphle, P. Wang, D. Jong, L. Wu, J. Lin et al., "Effects of aqueous extracts of "Betel Quid" and its constituents on testosterone production by dispersed mouse interstitial cells," American Journal of Chinese Medicine, vol. 32, no. 05, pp. 705-715, 2004.

[64] OECD, "Guideline for Testing of Chemicals, Acute Oral Toxicity- Acute Toxicity Class Method," 2001.

[65] L. L. Murphy and T. J. Lee, "Ginseng, sex behavior and nitric oxide," Annals of the New York Academy of Sciences, vol. 962, pp. 372-377, 2002.

[66] D. Cyranoski, "Malaysian researchers bet big on home-grown Viagra," Nature Medicine, vol. 11, article 912, 2005.

[67] H. H. Ang and M. K. Sim, "Eurycoma longifolia Jack enhances libido in sexually experienced male rats," Journal of Experimental Animal Science, vol. 46, no. 4, pp. 287-290, 1997.

[68] H. H. Ang and H. S. Cheang, "Effects of Eurycoma longifolia Jack on laevator ani muscle in both uncastrated and testosterone-stimulated castrated intact male rats," Archives of Pharmacal Research, vol. 24, no. 5, pp. 437-440, 2001. 

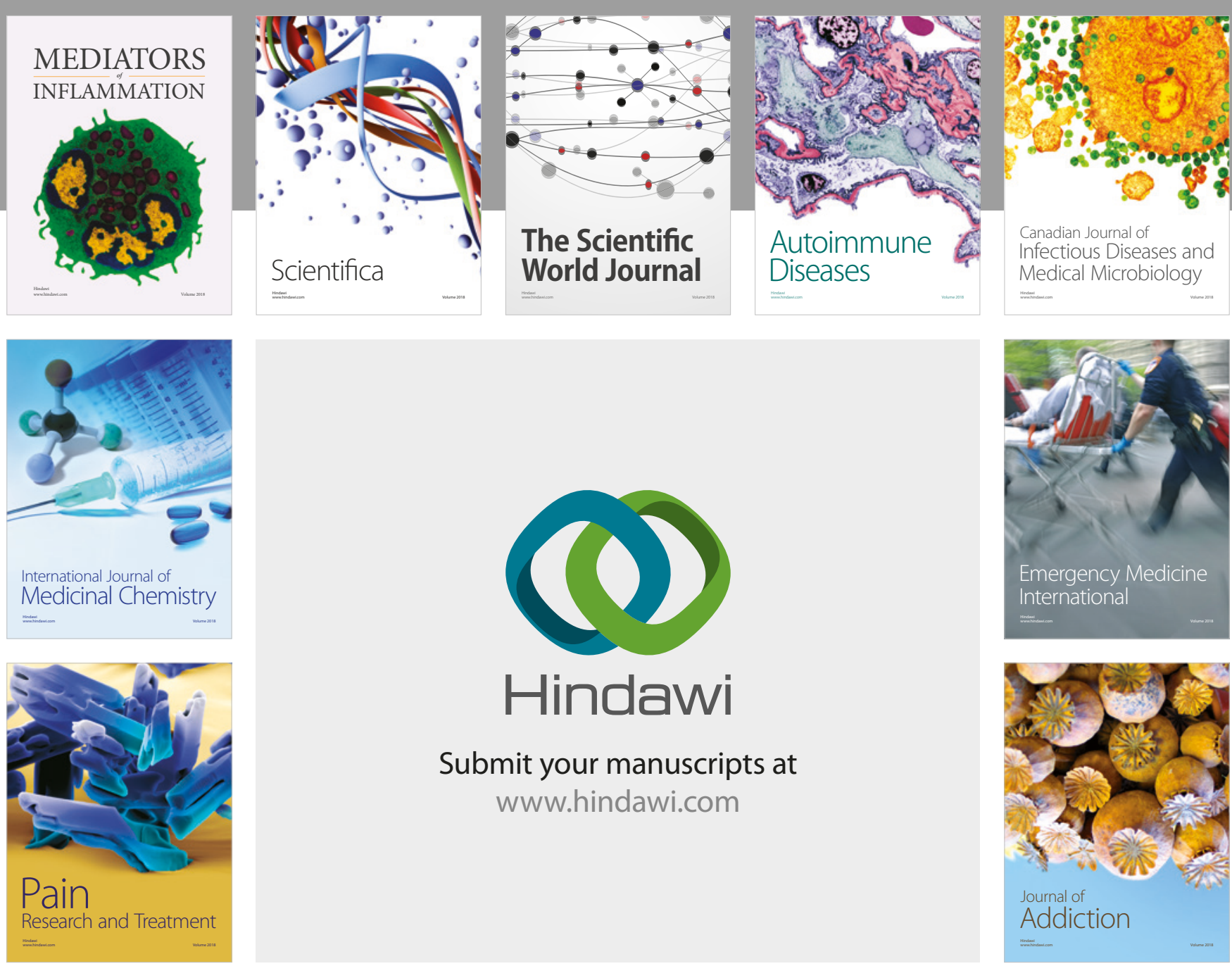

Canadian Journal of
Infectious Diseases and Medical Microbiology

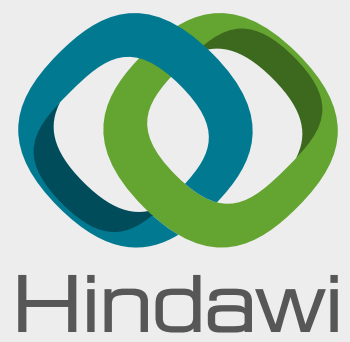

Submit your manuscripts at

www.hindawi.com
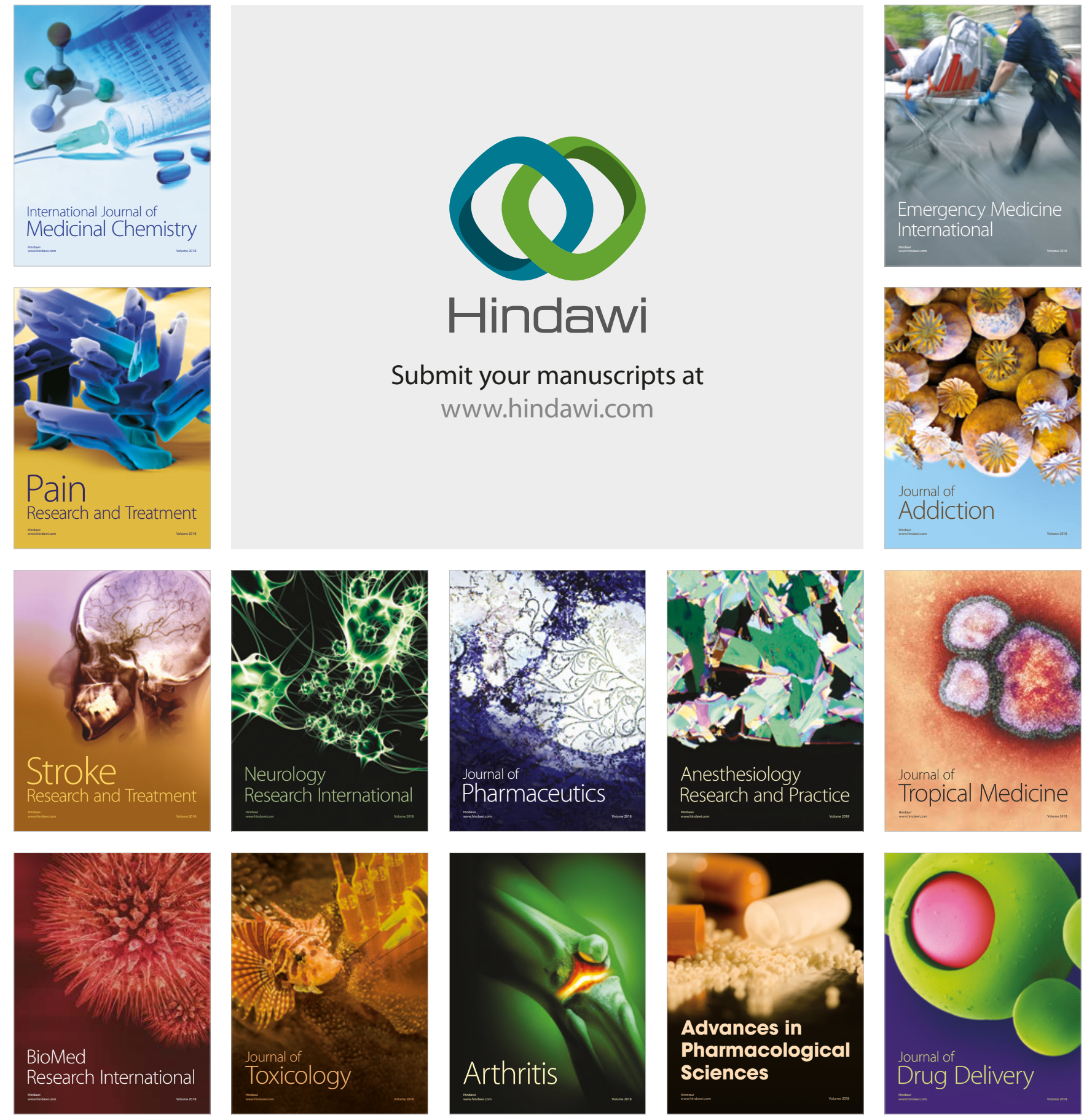